University of Nebraska - Lincoln

DigitalCommons@University of Nebraska - Lincoln

Agronomy \& Horticulture - Faculty Publications

Agronomy and Horticulture Department

November 2001

\title{
Predicting Leaf/Stem Ratio and Nutritive Value in Grazed and Nongrazed Big Bluestem
}

\author{
Alexander J. Smart \\ South Dakota State University \\ Walter H. Schacht \\ University of Nebraska-Lincoln, wschacht1@unl.edu \\ Lowell E. Moser \\ University of Nebraska-Lincoln, Imoser1@unl.edu
}

Follow this and additional works at: https://digitalcommons.unl.edu/agronomyfacpub

Part of the Plant Sciences Commons

Smart, Alexander J.; Schacht, Walter H.; and Moser, Lowell E., "Predicting Leaf/Stem Ratio and Nutritive Value in Grazed and Nongrazed Big Bluestem" (2001). Agronomy \& Horticulture -- Faculty Publications. 36.

https://digitalcommons.unl.edu/agronomyfacpub/36

This Article is brought to you for free and open access by the Agronomy and Horticulture Department at DigitalCommons@University of Nebraska - Lincoln. It has been accepted for inclusion in Agronomy \& Horticulture -Faculty Publications by an authorized administrator of DigitalCommons@University of Nebraska - Lincoln. 


\title{
FORAGES
}

\section{Predicting Leaf/Stem Ratio and Nutritive Value in Grazed and Nongrazed Big Bluestem}

\author{
Alexander J. Smart,* Walter H. Schacht, and Lowell E. Moser
}

\begin{abstract}
Recent advancements in describing morphological development of perennial grasses have provided a useful index for identifying dates to harvest hay or graze pasture. The objective of this study was to determine the relationship between big bluestem (Andropogon gerardii Vitman) morphological developmental stage, days from 1 May, and accumulated growing degree days (GDD) and leaf/stem ratio, crude protein (CP), and neutral detergent fiber (NDF) in grazed and nongrazed swards. The grazing experiment was conducted in eastern Nebraska during 1997 and 1998. Six grazing treatments consisting of all combinations of two herbage allowances $(22 \mathrm{or} 66 \mathrm{~kg}$ of herbage dry matter per animal unit day) and three grazing dates (late May, early June, and mid-June) were randomly assigned to seven paddocks $\left(900 \mathrm{~m}^{2}\right)$ in each of four blocks along with a nongrazed control treatment. Random samples consisting of $\mathbf{5 0}$ or more tillers were handclipped weekly at ground level in each paddock beginning in late May and ending in early August. Morphological developmental stage of the samples were determined, and a mean morphological stage based on shoot count [mean stage count (MSC)] was calculated. Samples were hand-separated to determine leaf/stem ratio and analyzed for CP and NDF. Regression analysis showed that good relationships were found between MSC $\left(R^{2}=0.61-0.81\right)$, days from 1 May $\left(R^{2}=\right.$ $0.45-0.76)$, and GDD $\left(R^{2}=0.44-0.74\right)$ and leaf/stem ratio in nongrazed big bluestem and high herbage allowance swards. All three were adequate predictors of nutritive value and leafiness, but MSC may be more useful to researchers who want a more descriptive measure of plant morphological development, particularly when comparing cultivars or species.
\end{abstract}

$\mathrm{M}$ ORPHOLOGICAL DEVELOPMENT OF FORAGE CROPS CAn be used to determine harvest date of alfalfa (Medicago sativa L.) (Kalu and Fick, 1981), predict forage quality in warm-season grasses (Mitchell et al., 2001), and grazing readiness of several cool-season grasses (Frank and Hofmann, 1989; Frank, 1991). Leaf/stem ratio is an important factor in determining quality, diet selection, and forage intake (Chacon and Stobbs, 1976; Chacon et al., 1978; Forbes and Coleman, 1993). Although leaf/ stem ratio is an important measurement in grazing studies and grass-breeding programs, grass swards are infrequently characterized in terms of it because estimating leaf/stem ratio is a tedious process of separating leaves and stems by hand. Information regarding the relationships between leaf/stem ratio and morphological devel-

Dep. of Agron. and Hortic., Univ. of Nebraska, 279 Plant Sci., Lincoln, NE 68583. A.J. Smart, current address: Dep. of Animal and Range Sci., Box 2170, South Dakota State Univ., Brookings, SD 57007-0392. Nebraska Agric. Exp. Stn. Journal Ser. no. 13206. Received 11 July 2000.*Corresponding author (smarta@sdstate.edu).

Published in Agron. J. 93:1243-1249 (2001). opment, day of year (DOY), and accumulated growing degree days (GDD) is lacking, and models developed to predict leaf/stem ratio would be useful.

The mean morphological stage based on shoot count [mean stage count (MSC)], using the system described by Kalu and Fick (1981), has been used successfully to predict forage quality parameters in alfalfa (Buxton et al., 1985; Buxton and Hornstein, 1986). Likewise, the system described by Simon and Park (1983) for quantifying the development of perennial grasses has been used to predict digestibility and crude protein $(\mathrm{CP})$ of coolseason grasses (Buxton and Marten, 1989). However, the samples used for determining developmental stages in these studies were not from a random sample of the population. Thus, an unbiased mean developmental stage was not determined. Alternatively, DOY and GDD have been used to predict forage quality in cool-season grasses (Buxton and Marten, 1989). Buxton and Marten (1989) noted that herbage age was more closely related to the spring decline in forage quality of cool-season grasses than was GDD or morphological development.

Recent advancement in quantifying the morphological development of perennial grasses (Moore et al., 1991) has provided a useful index with constant incremental changes among substages that allow evaluation of grass sward maturity based on a population mean instead of a few tillers. This system has been recommended by ASA, CSSA, and SSSA (Frank and Cardwell, 1997). Fick et al. (1994) proposed that this system might be more useful in predicting forage quality than age-quality relationships with species where developmental stage and quality respond to the environment in the same way.

Big bluestem, like other warm-season grasses, is determinant in growth habit, and its morphological development can be predicted by age and GDD (Mitchell et al., 1997). Plant maturity integrates cumulative effects of physiological processes that are expressed in yield and herbage quality (Buxton and Fales, 1994). Thus, morphological development, DOY, and GDD should predict leaf/stem ratio and forage quality parameters in big bluestem. Previous studies have concentrated on relationships between morphological development, DOY, or GDD and forage quality in nondefoliated swards (Buxton and Marten, 1989; Frank and Hofmann, 1989; Frank, 1991; Mitchell et al., 2001). Information is lacking on

Abbreviations: AUD, animal unit day; CP, crude protein; DOY, day of year; GDD, growing degree days; MSC, mean stage count; NDF, neutral detergent fiber. 
Table 1. Near-infrared reflectance calibration statistics for neutral detergent fiber (NDF) and crude protein (CP) prediction equations. $\dagger$

\begin{tabular}{|c|c|c|c|c|c|c|}
\hline Variable & $\mathbf{N}$ & Mean & SD§ & SECVII & SEC\# & $R^{2}$ \\
\hline & & & & $\mathbf{g}^{-1}$ & & \\
\hline NDF & 49 & 697 & 40.5 & 18.2 & 12.9 & 0.90 \\
\hline CP & 49 & 93 & 43.8 & 5.2 & 4.1 & 0.99 \\
\hline
\end{tabular}

$\dagger$ Number of samples in data set were 519, with 11 outliers removed. Math treatment was $1,4,4,1$ = derivative, gap, smooth, and smooth 2 .

+ Number of samples used in calibration equation.

$\S$ Standard deviation.

II Standard error of cross validation.

\# Standard error of calibration.

the relationships between morphological development, DOY, and GDD and forage quality characteristics on grazed swards. The objective of this study was to determine the relationship between big bluestem MSC, days from 1 May, and GDD and leaf/stem ratio, CP, and neutral detergent fiber (NDF) in grazed and nongrazed swards.

\section{MATERIALS AND METHODS}

A sward of 'Pawnee' big bluestem was established in a 4-ha field in 1995 and interseeded to thicken the sward in 1996 on a Sharpsburg silty clay loam (fine, montmorillonitic, mesic, Typic Argiudoll) at the Agricultural Research Development Center near Mead, NE. The sward was not fertilized during establishment or in pursuant years. Broadleaf weeds were controlled as needed by applying 2,4-D (2,4-dichlorophenoxy acetic acid) at $2.1 \mathrm{~L} \mathrm{ha}^{-1}$. The sward was not harvested in 1996, and considerable plant biomass accumulated. Prescribed burns were conducted in late April 1997 and 1998 to remove the dead plant material.

Plant material used in this study was collected in 1997 and 1998 from a grazing experiment conducted on this site and designed to determine yield responses of big bluestem following intensive early spring defoliation. The experiment was designed as a randomized complete block with four replicates. Six grazing treatments consisting of all combinations of two herbage allowances [22 or $66 \mathrm{~kg}$ of herbage dry matter per animal unit day (AUD, the herbage demand for $1 \mathrm{~d}$ for an animal weighing approximately $450 \mathrm{~kg}$ )] and three grazing dates (late-May, early-June, or mid-June) and a nongrazed control treatment were randomly assigned to seven paddocks $\left(900 \mathrm{~m}^{2}\right)$ in each replicate. In 1997, late May and early June grazing dates were pushed back to 2 and 9 June, respectively, because a late-spring freeze caused a delay in herbage growth. Yearling heifers (Bos taurus) grazed each paddock for $1 \mathrm{~d}$ at the determined grazing date and herbage allowance and then were removed.

Weekly herbage samples were collected from each paddock from early June through late July in 1997 and from late May through early August in 1998. Seven sample locations were selected randomly within each paddock. At each location, at least 50 tillers were hand-clipped at about $2.5 \mathrm{~cm}$ above ground level. Immediately following collection, all samples were brought to the laboratory, and the morphological developmental stage was determined (Moore et al., 1991). The MSC was calculated for each treatment at each sampling date. Two staged samples were dried in a forced-air oven at $60^{\circ} \mathrm{C}$ for $72 \mathrm{~h}$. Dried samples were hand-separated into leaf blade and stem (including leaf sheath and inflorescence) components and weighed to determine leaf/stem ratio. One sample of leaf/ stem ratio from each experimental unit was recombined and ground in a Wiley mill (Arthur Thomas Co., Philadelphia, PA) to pass a 1.0-mm screen and further ground through a cyclone mill (Udy Analyzer Co., Boulder, CO) with a 1.0-mm screen. Samples were stored in plastic bags at room temperature before near-infrared reflectance spectroscopy analysis was performed for $\mathrm{CP}$ and $\mathrm{NDF}$.

Ground forage samples were scanned using a Perstorp model 6500 near-infrared scanning monochromator, and software options Center and Select (NIRSystems, Perstorp Analytical Co., Silver Spring, MD) were used for closed model calibration equation development. Wet chemistry procedures using the ANKOM Fiber Analyzer (ANKOM 200, Ankom Technol., Fairport, NY) described by Vogel et al. (1999) were used to determine NDF. Nitrogen concentration was determined with a FP-428 N determinator system (601-700-300, LECO Corp., St. Joseph, MI). Crude protein content was calculated as percentage $\mathrm{N} \times 6.25$. Calibration statistics for NDF and CP prediction equations are shown in Table 1.

Multiple regression analysis was performed on experimental-unit means using PROC REG with STEPWISE model selection and $\alpha=0.15$ for variable entry criteria (SAS, 1990)

Table 2. Range of leaf/stem ratio, crude protein (CP), and neutral detergent fiber (NDF) of big bluestem for grazing treatments in 1997 and 1998 at Mead, NE.

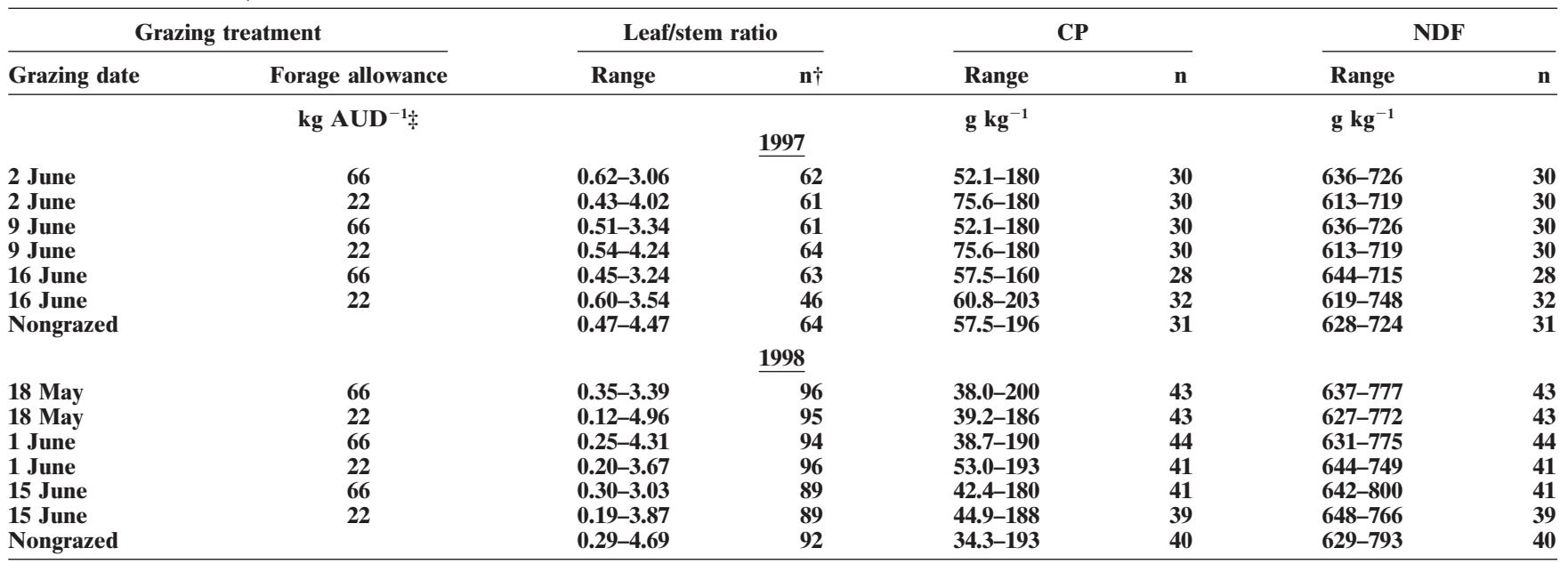

$\dagger$ Number of samples.

\$ AUD, animal unit day. 
to model leaf/stem ratio, $\mathrm{CP}$, and NDF from MSC, days from 1 May, and GDD. Accumulated GDD were calculated using the following formula for each year:

$$
\begin{gathered}
\mathrm{GDD}= \\
\sum(\text { from } 1 \text { May to sampling date }) \\
{\left[\left(T_{\max }+T_{\min }\right) / 2\right]-5^{\circ} \mathrm{C}}
\end{gathered}
$$

where $T_{\max }$ and $T_{\min }$ are the daily maximum and minimum temperatures, respectively, in ${ }^{\circ} \mathrm{C}$.

\section{RESULTS AND DISCUSSION Leaf/Stem Ratio}

Leaf/stem ratio had a larger range in 1998 than in 1997 (Table 2). The 1998 sampling period extended into August when big bluestem herbage was composed of many reproductive culms with less leaf biomass. Vegetation sampling began late in 1997 due to a late-spring freeze, which delayed the initial spring grazing treatment by 2 wk until 2 June.
In 1997, MSC accounted for $81 \%$ of the variation in leaf/stem ratio in the nongrazed swards and in the swards grazed in late May at the $66 \mathrm{~kg} \mathrm{AUD}^{-1}$ herbage allowance. In 1998, MSC accounted for 73 and $76 \%$ of the variation in leaf/stem ratio in the nongrazed swards and late May grazed swards at the $66 \mathrm{~kg} \mathrm{AUD}^{-1}$ herbage allowance, respectively. Predictions of leaf/stem ratio were not as good for the last two grazing dates at the high herbage allowance during either year (Table 3 ). Values of coefficient of determination and root mean square error were low and high, respectively, for the regression equations relating MSC to leaf/stem ratio for big bluestem in swards grazed at the $22 \mathrm{~kg} \mathrm{AUD}^{-1}$ herbage allowance on any of the three grazing dates. The general predicted response of leaf/stem ratio to increasing MSC was a quadratic decline for the nongrazed and high herbage allowance treatments, whereas the low forage allowance treatments under-predicted leaf/stem ratio at both low MSC and high MSC values

Table 3. Leaf/stem ratio (LS) prediction for big bluestem from mean stage count (MSC), days from 1 May (DAY), and accumulated

\begin{tabular}{|c|c|c|c|c|}
\hline \multicolumn{2}{|c|}{ Grazing treatment } & \multirow[b]{2}{*}{ Regression equation } & \multirow[b]{2}{*}{$\mathbf{R M S E} \dagger$} & \multirow{2}{*}{$\begin{array}{l}\text { Coefficient of } \\
\text { determination }\end{array}$} \\
\hline Grazing date & Forage allowance & & & \\
\hline \multicolumn{5}{|c|}{ kg AUD ${ }^{-1}+$} \\
\hline \multicolumn{5}{|c|}{1997} \\
\hline 2 June & 66 & $\mathrm{LS}=7.1-4.4(\mathrm{MSC})+0.69\left(\mathrm{MSC}^{2}\right)$ & 0.286 & 0.81 \\
\hline 2 June & 22 & $\mathbf{L S}=-1.4+5.7\left(\mathrm{MSC}^{\prime}\right)-2.3\left(\mathrm{MSC}^{2}\right)$ & 0.646 & 0.41 \\
\hline 9 June & 66 & $\mathbf{L S}=13-12(\mathrm{MSC})+2.9\left(\mathrm{MSC}^{2}\right)$ & 0.418 & 0.61 \\
\hline 9 June & 22 & $\mathrm{LS}=\mathbf{4 . 0}-\mathbf{1 . 5}(\mathrm{MSC})$ & 0.598 & 0.36 \\
\hline 16 June & 66 & $\mathbf{L S}=9.2-7.1(\mathrm{MSC})+1.5\left(\mathrm{MSC}^{2}\right)$ & 0.374 & 0.69 \\
\hline 16 June & 22 & LS $=-6.4+11\left(\mathrm{MSC}^{2}-4.1\left(\mathrm{MSC}^{2}\right)\right.$ & 0.550 & 0.21 \\
\hline Nongrazed & & $\mathbf{L S}=10.3-7.8\left(\mathrm{MSC}^{\prime}\right)+1.5\left(\mathrm{MSC}^{2}\right)$ & 0.365 & 0.81 \\
\hline 2 June & 66 & $\mathbf{L S}=1.2+0.061(\mathrm{DA} Y)-0.00079\left(\mathrm{DA} \mathbf{Y}^{2}\right)$ & 0.346 & 0.75 \\
\hline 2 June & 22 & $\mathbf{L S}=0.3+0.094(\mathrm{DAY})-0.0010\left(\mathrm{DAY}^{2}\right)$ & 0.638 & 0.42 \\
\hline 9 June & 66 & $\mathbf{L S}=2.7-0.00022\left(\mathrm{DAY}^{2}\right)$ & 0.492 & 0.45 \\
\hline 9 June & 22 & $\mathbf{L S}=3.6-0.028(\mathrm{DAY})$ & 0.590 & 0.37 \\
\hline 16 June & 66 & $\mathbf{L S}=3.8-\mathbf{0 . 0 3 5}(\mathrm{DAY})$ & 0.358 & 0.71 \\
\hline 16 June & 22 & $\mathbf{L S}=1.9-0.000086\left(\mathrm{DAY}^{2}\right)$ & 0.593 & $\mathbf{0 . 0 5}$ \\
\hline Nongrazed & & $\mathbf{L S}=3.18-0.00036\left(\mathrm{DAY}^{2}\right)$ & 0.434 & 0.72 \\
\hline 2 June & 66 & $\mathbf{L S}=2.0+0.0016(G D D)-0.0000021\left(G^{2} D^{2}\right)$ & 0.330 & 0.74 \\
\hline 2 June & 22 & $\mathbf{L S}=1.6+\mathbf{0 . 0 0 3 2}(\mathrm{GDD})-\mathbf{0 . 0 0 0 0 0 3 0}\left(\mathrm{GDD}^{2}\right)$ & 0.639 & 0.38 \\
\hline 9 June & 66 & $\mathbf{L S}=2.4-0.00000089\left(\mathbf{G D D}^{2}\right)$ & 0.496 & 0.44 \\
\hline 9 June & 22 & LS $=2.9-0.0015($ GDD $)$ & 0.594 & 0.37 \\
\hline 16 June & 66 & LS $=3.2-0.0019($ GDD $)$ & 0.358 & 0.71 \\
\hline 16 June & 22 & LS $=1.8-0.00000034\left(G^{2} D^{2}\right)$ & 0.591 & 0.06 \\
\hline Nongrazed & & $\mathbf{L S}=2.8-0.0000014\left(G^{2} D^{2}\right)$ & 0.440 & 0.72 \\
\hline \multicolumn{5}{|c|}{$\underline{1998}$} \\
\hline 18 May & 66 & $\mathrm{LS}=9.2-7.0(\mathrm{MSC})+1.4\left(\mathrm{MSC}^{2}\right)$ & 0.421 & 0.73 \\
\hline 18 May & 22 & $\mathbf{L S}=\mathbf{7 . 7}-\mathbf{5 . 2}(\mathrm{MSC})+\mathbf{0 . 8 8}\left(\mathrm{MSC}^{2}\right)$ & 0.667 & 0.60 \\
\hline 1 June & 66 & $\mathbf{L S}=8.2-6.2(\mathrm{MSC})+1.2\left(\mathrm{MSC}^{2}\right)$ & 0.521 & 0.59 \\
\hline 1 June & 22 & $\mathbf{L S}=2.6-0.45\left(\mathrm{MSC}^{2}\right)$ & 0.695 & 0.27 \\
\hline 15 June & 66 & $\mathbf{L S}=8.8-6.7(\mathrm{MSC})+1.4\left(\mathrm{MSC}^{2}\right)$ & 0.428 & 0.69 \\
\hline 15 June & 22 & $\mathbf{L S}=2.7-0.47\left(\mathbf{M S C}^{2}\right)$ & 0.706 & 0.27 \\
\hline Nongrazed & & $\mathbf{L S}=8.5-6.1(\mathrm{MSC})+1.1\left(\mathrm{MSC}^{2}\right)$ & 0.446 & 0.76 \\
\hline 18 May & 66 & $\mathbf{L S}=2.05+0.019(\mathrm{DAY})-0.00039\left(\mathrm{DAY}^{2}\right)$ & 0.473 & 0.67 \\
\hline 18 May & 22 & $\mathbf{L S}=1.0+0.073(\mathrm{DAY})-0.00088\left(\mathrm{DAY} \mathbf{Y}^{2}\right)$ & 0.664 & 0.60 \\
\hline 1 June & 66 & $\mathbf{L S}=3.0-0.025(\mathrm{DAY})$ & 0.531 & $\mathbf{0 . 5 7}$ \\
\hline 1 June & 22 & $\mathbf{L S}=2.7-0.019(\mathrm{DAY})$ & 0.665 & $\mathbf{0 . 3 3}$ \\
\hline 15 June & 66 & $\mathbf{L S}=\mathbf{2 . 5}-\mathbf{0 . 0 0 0 2 3}\left(\mathrm{DA} \mathbf{Y}^{2}\right)$ & 0.430 & 0.68 \\
\hline 15 June & 22 & $\mathbf{L S}=\mathbf{2 . 3}-0.00017\left(\mathrm{DA} \mathbf{Y}^{2}\right)$ & 0.672 & 0.34 \\
\hline Nongrazed & & $\mathbf{L S}=2.7-0.00027\left(\mathrm{DAY}^{2}\right)$ & 0.473 & 0.72 \\
\hline 18 May & 66 & $\mathbf{L S}=2.4-0.00000081\left(G^{2} D^{2}\right)$ & 0.463 & 0.68 \\
\hline 18 May & 22 & $\mathbf{L S}=1.7+0.0026($ GDD $)-0.0000023\left(G^{2} D^{2}\right)$ & 0.666 & 0.60 \\
\hline 1 June & 66 & LS $=2.8-0.0014($ GDD $)$ & 0.532 & 0.55 \\
\hline 1 June & 22 & $\mathbf{L S}=2.5-0.0010($ GDD $)$ & 0.672 & 0.31 \\
\hline 15 June & 66 & $\mathbf{L S}=2.4-0.00000081\left(G^{\prime} D^{2}\right)$ & 0.417 & 0.70 \\
\hline 15 June & 22 & $\mathbf{L S}=2.2-0.00000061\left(G^{2} D^{2}\right)$ & 0.662 & 0.35 \\
\hline Nongrazed & & $\mathbf{L S}=3.2-0.0018($ GDD $)$ & 0.475 & 0.74 \\
\hline
\end{tabular}
growing degree days. (GDD) for grazing treatments in 1997 and 1998 at Mead, NE.

$\dagger$ Root mean square error.

+ AUD, animal unit day. 


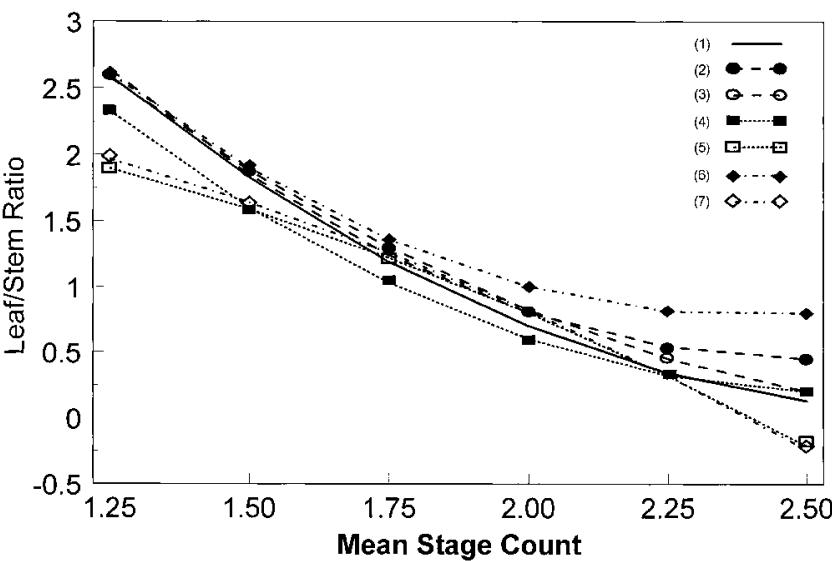

Fig. 1. Leaf/stem ratio predictions by mean stage count (MSC) of big bluestem in eastern Nebraska in 1998 on (1) nongrazed swards and swards grazed for $1 \mathrm{~d}$, (2) 18 May at $66 \mathrm{~kg}$ per animal unit day (AUD, the herbage demand for $1 \mathrm{~d}$ from an animal weighing approximately $450 \mathrm{~kg}$ ), (3) 18 May at $22 \mathrm{~kg} \mathrm{AUD}^{-1}$, (4) 1 June at $66 \mathrm{~kg} \mathrm{AUD}^{-1}$, (5) 1 June at $22 \mathrm{~kg} \mathrm{AUD}^{-1}$, (6) 15 June at $66 \mathrm{~kg}$ AUD $^{-1}$, and (7) 15 June at $22 \mathrm{~kg} \mathrm{AUD}^{-1}$.
(Fig. 1). Predicted leaf/stem ratio for the low herbage allowance treatments from the last two grazing dates declined as MSC increased because leaf/stem ratio was relatively low due to intense defoliation of the grazing treatment (Fig. 1). Thus, MSC could only be used to predict leaf/stem ratio of big bluestem tillers that were not defoliated or only lightly defoliated early in the growing season. Mean stage count should have been a good predictor of leaf/stem ratio in the nongrazed control because the proportion of stem in grass plants increases as they mature or progress from the vegetative to reproductive stage (Mitchell et al., 1997). Mean stage count is an arithmetic average of individual tillers at particular morphological stages and does not account for differences in tiller size. Developmental stage expressed as a mean stage by weight may better predict leaf/stem ratio because it is a weighted mean based on tiller mass rather than count. Results also indicated that the relation between MSC and leaf/stem ratio was largely unchanged in grazing paddocks at low levels of utilization, especially early in the growing season. However,

Table 4. Crude protein (CP) prediction for big bluestem from mean stage count (MSC), days from 1 May (DAY), and accumulated growing degree days (GDD) for grazing treatments in 1997 and 1998 at Mead, NE.

\begin{tabular}{|c|c|c|c|c|}
\hline \multicolumn{2}{|c|}{ Grazing treatment } & \multirow[b]{2}{*}{ Regression equation } & \multirow[b]{2}{*}{$\mathbf{R M S E} \dagger$} & \multirow{2}{*}{$\begin{array}{l}\text { Coefficient of } \\
\text { determination }\end{array}$} \\
\hline Grazing date & Forage allowance & & & \\
\hline \multicolumn{4}{|c|}{ 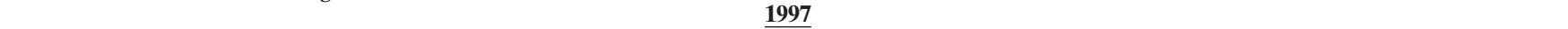 } & \\
\hline $\begin{array}{l}2 \text { June } \\
2 \text { June } \\
9 \text { June } \\
9 \text { June } \\
16 \text { June } \\
16 \text { June } \\
\text { Nongrazed }\end{array}$ & $\begin{array}{l}66 \\
22 \\
66 \\
22 \\
66 \\
22\end{array}$ & 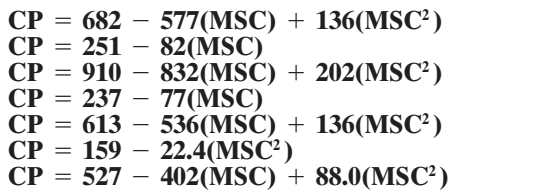 & $\begin{array}{l}19.76 \\
20.46 \\
17.67 \\
24.78 \\
24.44 \\
20.12 \\
19.74\end{array}$ & $\begin{array}{l}0.74 \\
0.58 \\
0.72 \\
0.50 \\
0.48 \\
0.33 \\
0.69\end{array}$ \\
\hline $\begin{array}{l}2 \text { June } \\
2 \text { June } \\
9 \text { June } \\
9 \text { June } \\
16 \text { June } \\
16 \text { June } \\
\text { Nongrazed }\end{array}$ & $\begin{array}{l}66 \\
22 \\
66 \\
22 \\
66 \\
22\end{array}$ & 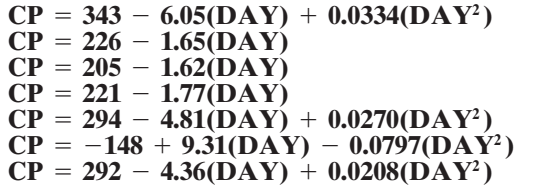 & $\begin{array}{l}13.29 \\
15.58 \\
21.24 \\
19.05 \\
20.03 \\
17.57 \\
15.74\end{array}$ & $\begin{array}{l}0.88 \\
0.76 \\
0.57 \\
0.71 \\
0.65 \\
0.51 \\
0.80\end{array}$ \\
\hline $\begin{array}{l}2 \text { June } \\
2 \text { June } \\
9 \text { June } \\
9 \text { June } \\
16 \text { June } \\
16 \text { June } \\
\text { Nongrazed }\end{array}$ & $\begin{array}{l}66 \\
22 \\
66 \\
22 \\
66 \\
22\end{array}$ & 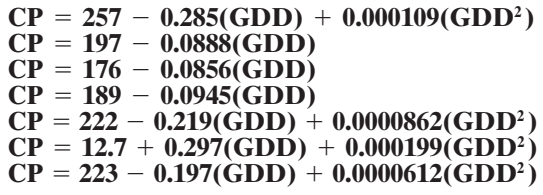 & $\begin{array}{l}12.91 \\
15.44 \\
21.30 \\
19.30 \\
20.31 \\
17.59 \\
16.15\end{array}$ & $\begin{array}{l}0.89 \\
0.76 \\
0.57 \\
0.70 \\
0.63 \\
0.51 \\
0.79\end{array}$ \\
\hline \multicolumn{5}{|c|}{$\underline{1998}$} \\
\hline $\begin{array}{l}18 \text { May } \\
18 \text { May } \\
1 \text { June } \\
1 \text { June } \\
15 \text { June } \\
15 \text { June } \\
\text { Nongrazed }\end{array}$ & $\begin{array}{l}66 \\
22 \\
66 \\
22 \\
66 \\
22\end{array}$ & $\begin{array}{l}\mathrm{CP}=\mathbf{8 8 6}-\mathbf{8 5 2}(\mathrm{MSC})+\mathbf{2 1 4}\left(\mathrm{MSC}^{2}\right) \\
\mathrm{CP}=\mathbf{4 5 1}-\mathbf{3 4 4}(\mathrm{MSC})+\mathbf{7 1 . 9}\left(\mathrm{MSC}^{2}\right) \\
\mathrm{CP}=\mathbf{6 4 2}-\mathbf{5 8 7}(\mathrm{MSC})+\mathbf{1 4 1}\left(\mathrm{MSC}^{2}\right) \\
\mathbf{C P}=\mathbf{1 8 6}-\mathbf{6 3 . 5}(\mathrm{MSC}) \\
\mathbf{C P}=\mathbf{5 4 9}-\mathbf{4 8 0 ( M S C )}+\mathbf{1 1 3}\left(\mathrm{MSC}^{2}\right) \\
\mathrm{CP}=\mathbf{2 0 8}-\mathbf{8 5 . 1 ( M S C )} \\
\mathbf{C P}=\mathbf{6 9 6}-\mathbf{6 2 3 ( M S C )}+\mathbf{1 4 7}\left(\mathrm{MSC}^{2}\right)\end{array}$ & $\begin{array}{l}18.76 \\
19.85 \\
23.45 \\
30.68 \\
22.99 \\
29.79 \\
19.92\end{array}$ & $\begin{array}{l}\mathbf{0 . 8 0} \\
\mathbf{0 . 7 7} \\
\mathbf{0 . 6 5} \\
\mathbf{0 . 2 0} \\
\mathbf{0 . 5 9} \\
\mathbf{0 . 3 1} \\
\mathbf{0 . 7 7}\end{array}$ \\
\hline $\begin{array}{l}18 \text { May } \\
18 \text { May } \\
1 \text { June } \\
1 \text { June } \\
15 \text { June } \\
15 \text { June } \\
\text { Nongrazed }\end{array}$ & $\begin{array}{l}66 \\
22 \\
66 \\
22 \\
66 \\
22\end{array}$ & 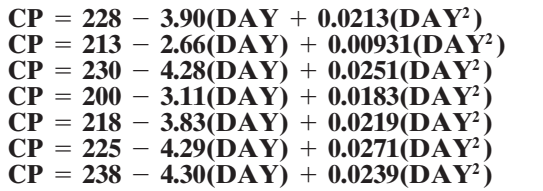 & $\begin{array}{r}14.06 \\
10.85 \\
12.64 \\
21.49 \\
12.88 \\
16.42 \\
9.13\end{array}$ & $\begin{array}{l}0.88 \\
0.93 \\
0.90 \\
0.62 \\
0.87 \\
0.80 \\
0.95\end{array}$ \\
\hline $\begin{array}{l}18 \text { May } \\
18 \text { May } \\
1 \text { June } \\
1 \text { June } \\
15 \text { June } \\
15 \text { June } \\
\text { Nongrazed }\end{array}$ & $\begin{array}{l}66 \\
22 \\
66 \\
22 \\
66 \\
22\end{array}$ & 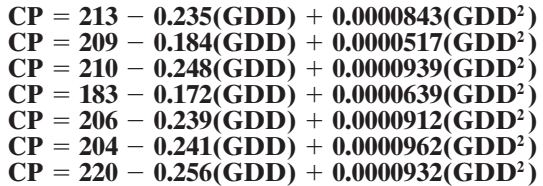 & $\begin{array}{l}14.65 \\
11.20 \\
14.16 \\
22.02 \\
14.93 \\
17.62 \\
10.70\end{array}$ & $\begin{array}{l}0.88 \\
0.93 \\
0.87 \\
0.59 \\
0.84 \\
0.78 \\
0.94\end{array}$ \\
\hline
\end{tabular}

$\dagger$ Root mean square error.

+ AUD, animal unit day. 
MSC became an increasingly poor predictor of leaf/ stem ratio as the grazing date advanced further into the growing season at $22 \mathrm{~kg} \mathrm{AUD}^{-1}$ herbage allowance.

Plants were vegetative at all grazing dates, and defoliation heights were generally above the apical meristem. Grazing did not affect MSC of grazed vs. nongrazed vegetative tillers because leaves were counted regardless of their level of defoliation. However, grazing did affect leaf/stem ratio, especially at the lower herbage allowance treatments because significant amounts of leaf blade tissue were removed. Severely defoliated tillers had relatively low leaf blade mass for several weeks that did not fully recover before culm elongation. Thus, MSC did not accurately reflect leaf/stem ratio for much of the season. The lightly grazed paddocks had less leaf blade mass removed and increased similarly in sward maturity and biomass accumulation compared with the nongrazed sward.
Values of root mean square error and coefficient of determination for regression equations using days from 1 May or GDD to predict leaf/stem ratio in the different grazing treatments were similar to MSC equations ( $\mathrm{Ta}$ ble 3). Order of polynomials were identical for days from 1 May and GDD equations in 1997 and similar in 1998.

\section{Nutritive Value}

Crude protein and NDF had a larger range in 1998 than in 1997 (Table 2). In 1997, the range in CP was greater for the nongrazed control and the paddocks grazed at the high herbage allowance than for paddocks grazed at the low herbage allowance, whereas the grazing treatments had similar ranges in 1998. Lower CP values in 1998 were a result of samples collected later in the summer than in 1997.

Table 5. Neutral detergent fiber (NDF) prediction for big bluestem from mean stage count (MSC), days from 1 May (DAY), and accumulated growing degree days (GDD) for grazing treatments in 1997 and 1998 at Mead, NE.

\begin{tabular}{|c|c|c|c|c|}
\hline \multicolumn{2}{|c|}{ Grazing treatment } & \multirow[b]{2}{*}{ Regression equation } & \multirow[b]{2}{*}{ RMSE $\dagger$} & \multirow{2}{*}{$\begin{array}{l}\text { Coefficient of } \\
\text { determination }\end{array}$} \\
\hline Grazing date & Forage allowance & & & \\
\hline \multicolumn{5}{|c|}{ kg AUD ${ }^{-1} \ddagger$} \\
\hline \multicolumn{5}{|c|}{$\underline{1997}$} \\
\hline 2 June & 66 & $\mathrm{NDF}=622+17.8(\mathrm{MSC})$ & 12.68 & 0.70 \\
\hline 2 June & 22 & $\mathrm{NDF}=632+13.1\left(\mathrm{MSC}^{2}\right)$ & 20.83 & 0.27 \\
\hline 9 June & 66 & $\mathrm{NDF}=435+238(\mathrm{MSC})-52.6\left(\mathrm{MSC}^{2}\right)$ & 12.37 & 0.54 \\
\hline 9 June & 22 & $\mathrm{NDF}=626+17.7\left(\mathrm{MSC}^{2}\right)$ & 19.23 & 0.51 \\
\hline 16 June & 66 & $\mathrm{NDF}=592+47.1(\mathrm{MSC})$ & 17.11 & 0.41 \\
\hline 16 June & 22 & $\mathrm{NDF}=636+15.0\left(\mathrm{MSC}^{2}\right)$ & 20.65 & 0.17 \\
\hline Nongrazed & & $\mathrm{NDF}=632+14.3\left(\mathrm{MSC}^{2}\right)$ & 15.33 & 0.58 \\
\hline 2 June & 66 & NDF $=609+1.008($ DAY $)$ & 14.79 & 0.59 \\
\hline 2 June & 22 & $\mathrm{NDF}=621+0.702(\mathrm{DAY})$ & 21.42 & 0.23 \\
\hline 9 June & 66 & $\mathrm{NDF}=628+0.833(\mathrm{DAY})$ & 12.49 & 0.51 \\
\hline 9 June & 22 & $\mathrm{NDF}=606+1.11(\mathrm{DAY})$ & 20.32 & 0.45 \\
\hline 16 June & 66 & $\mathrm{NDF}=611+0.989(\mathrm{DAY})$ & 15.97 & 0.48 \\
\hline 16 June & 22 & $\mathrm{NDF}=621+0.726(\mathrm{DAY})$ & 20.88 & 0.15 \\
\hline Nongrazed & & $\mathrm{NDF}=618+0.935(\mathrm{DAY})$ & 18.11 & 0.41 \\
\hline 2 June & 66 & $\mathrm{NDF}=647+0.0000368\left(\mathrm{GDD}^{2}\right)$ & 14.52 & 0.61 \\
\hline 2 June & 22 & NDF $=682-0.109($ GDD $)+0.0000944\left(G^{\prime} D^{2}\right)$ & 20.03 & 0.35 \\
\hline 9 June & 66 & NDF $=659+0.0000269\left(\right.$ GDD $\left.^{2}\right)$ & 12.29 & 0.52 \\
\hline 9 June & 22 & NDF $=677-0.0967($ GDD $)+0.000100\left(G^{2} D^{2}\right)$ & 18.70 & 0.55 \\
\hline 16 June & 66 & NDF $=647+0.0000324\left(G^{2} D^{2}\right)$ & 15.81 & 0.48 \\
\hline 16 June & 22 & $\mathrm{NDF}=818-0.398(G D D)+0.000244\left(G^{\prime} D D^{2}\right)$ & 16.99 & 0.47 \\
\hline Nongrazed & & NDF $=651+0.0000326\left(G^{2} D^{2}\right)$ & 17.47 & 0.45 \\
\hline \multicolumn{5}{|c|}{$\underline{1998}$} \\
\hline 18 May & 66 & $\mathrm{NDF}=220+46 \overline{(\mathrm{MSC})}-98.3\left(\mathrm{MSC}^{2}\right)$ & 18.43 & 0.80 \\
\hline 18 May & 22 & $\mathrm{NDF}=376+292(\mathrm{MSC})-\mathbf{5 4 . 2 ( \mathrm { MSC } ^ { 2 } )}$ & 14.11 & 0.89 \\
\hline 1 June & 66 & $\mathrm{NDF}=287+421(\mathrm{MSC})-93.5\left(\mathrm{MSC}^{2}\right)$ & 19.67 & 0.72 \\
\hline 1 June & 22 & $\mathrm{NDF}=\mathbf{5 9 5}+\mathbf{7 0 . 5 ( \mathrm { MSC } )}$ & 20.94 & 0.40 \\
\hline 15 June & 66 & $\mathrm{NDF}=\mathbf{5 6 8}+\mathbf{8 7 . 7 ( \mathrm { MSC } )}$ & 26.28 & 0.45 \\
\hline 15 June & 22 & $\mathrm{NDF}=911-324(\mathrm{MSC})+123\left(\mathrm{MSC}^{2}\right)$ & 25.78 & 0.30 \\
\hline Nongrazed & & $\mathrm{NDF}=312+352(\mathrm{MSC})-67.3\left(\mathrm{MSC}^{2}\right)$ & 18.26 & 0.85 \\
\hline 18 May & 66 & $\mathrm{NDF}=653+0.0128\left(\mathrm{DAY}^{2}\right)$ & 15.87 & 0.85 \\
\hline 18 May & 22 & $\mathrm{NDF}=641+0.0141\left(\mathrm{DAY} \mathbf{Y}^{2}\right)$ & 11.78 & 0.92 \\
\hline 1 June & 66 & NDF $=634+1.32($ DAY $)$ & 16.40 & 0.80 \\
\hline 1 June & 22 & $\mathrm{NDF}=671+0.00722\left(\mathrm{DAY}^{2}\right)$ & 17.94 & 0.56 \\
\hline 15 June & 66 & $\mathrm{NDF}=623+1.50(\mathrm{DAY})$ & 18.38 & 0.78 \\
\hline 15 June & 22 & $\mathrm{NDF}=620+2.25(\mathrm{DAY})-0.0108\left(\mathrm{DAY}^{2}\right)$ & 17.57 & 0.67 \\
\hline Nongrazed & & $\mathrm{NDF}=606+1.74(\mathrm{DAY})$ & 15.74 & 0.88 \\
\hline 18 May & 66 & $\mathrm{NDF}=630+\mathbf{0 . 0 8 3 0}($ GDD $)$ & 15.94 & 0.85 \\
\hline 18 May & 22 & $\mathrm{NDF}=649+0.0000482\left(\mathrm{GDD}^{2}\right)$ & 11.72 & 0.92 \\
\hline 1 June & 66 & NDF $=646+0.0725($ GDD $)$ & 16.64 & 0.79 \\
\hline 1 June & 22 & $\mathrm{NDF}=659+0.0458($ GDD $)$ & 17.86 & 0.55 \\
\hline 15 June & 66 & NDF $=638+0.0810($ GDD $)$ & 18.68 & 0.76 \\
\hline 15 June & 22 & $\mathrm{NDF}=624+0.148(\mathrm{GDD})-0.0000499\left(\mathrm{GDD}^{2}\right)$ & 17.56 & 0.69 \\
\hline Nongrazed & & $\mathrm{NDF}=621+0.0960($ GDD $)$ & 15.69 & 0.88 \\
\hline
\end{tabular}

$\dagger$ Root mean square error.

$\$$ AUD, animal unit day. 
In 1997 and 1998, MSC was more effective for predicting $\mathrm{CP}$ in the high herbage allowance treatments at the first two grazing dates and the nongrazed treatment than low herbage allowance treatments at all grazing dates (Table 4). Defoliated tillers from the low herbage allowance treatment may have the same MSC as nondefoliated tillers, but whole-plant forage quality would be altered due to less leaf mass. Prediction of NDF from MSC was poor in 1997 for all treatments except the 2 June grazing date at high herbage allowance (Table 5). However, in 1998, MSC accurately predicted NDF in the nongrazed treatment, grazed treatments on 18 May at both herbage allowances, and the grazed treatment on 1 June at the high herbage allowance. The differences in accuracy of regression equations between years might have been due to the longer vegetation sampling period in 1998 than in 1997. Ranges in CP and NDF were more similar for all treatments in 1998 than in 1997 (Table 2 ). Thus, inclusion of samples that represented more mature herbage may have helped increase the accuracy in the low herbage allowance grazing treatments.

Values of coefficient of determination and root mean square error were similar between days from 1 May and GDD for all treatments predicting CP and NDF in 1997 and 1998 (Tables 4 and 5). Regression equations were generally more accurate in 1998 than in 1997. Prediction of NDF in big bluestem treatments by days from 1 May and GDD in our study were better than predictions of NDF by DOY in grazed ( $r^{2}$ ranged from $0.58-0.67$ ) and nongrazed $\left(r^{2}\right.$ ranged from $\left.0.30-0.69\right)$ switchgrass (Panicum virgatum L.) (Gabrielsen et al., 1990). Mitchell et al. (2001) found that whole-plant CP in nondefoliated big bluestem was explained best by GDD $\left(R^{2}=\right.$ $0.96)$, followed by DOY $\left(R^{2}=0.92\right)$ and MSC $\left(R^{2}=\right.$ $0.83)$. They also found that whole-plant NDF was predicted best by $\operatorname{MSC}\left(R^{2}=0.75\right)$, followed by GDD $\left(R^{2}=\right.$ $0.70)$ and DOY $\left(r^{2}=0.52\right)$. In this study, days from 1 May and GDD equations were generally better than MSC equations in predicting CP (Table 4). However, MSC equations were better than days from 1 May and GDD equations in predicting NDF (Table 5), which is consistent with results from Mitchell et al. (2001).

Disturbance to the sward structure caused by low herbage allowance resulted in poor predictions of leaf/ stem ratio, CP, and NDF, especially when compared with nongrazed or lightly, early defoliated swards. Good relationships between MSC, days from 1 May, or GDD and $\mathrm{CP}$ and NDF in nongrazed big bluestem swards were similar to findings reported by others on nondefoliated grasses. Days from 1 May and GDD were better predictors of CP than MSC; however, MSC predicted leaf/stem ratio and NDF with equal or better accuracy than days from 1 May or GDD in grazed swards. The accuracy of predicting leaf/stem ratio and nutritive values on heavily grazed swards using the independent variables measured in this study was low. Improvements in prediction accuracy likely would be made by identifying other independent variables related to measures of defoliation intensity, such as percentage of leaf remaining or sward height reduction, and including these variables in a model with MSC, days from 1 May, or GDD.

Calendar day and GDD have been used to accurately predict nutritive value in perennial grasses and annual cereal grasses and were usually better predictors than morphological developmental stage (Buxton and Marten, 1989; Mitchell et al., 2001; Sanderson and Wedin, 1989; West et al., 1991). However, Buxton and Marten (1989) noted that calendar day and GDD would not be useful in explaining differences in leafiness and nutritive value between cultivars within a single species because cultivars would respond differently to environments. Nevertheless, calendar day and GDD, supported by this research and others, are adequate predictors of nutritive value and leafiness for producers planning haying schedules or grazing turnout dates. Mean stage count is useful to researchers who want a more descriptive measure of plant morphological development, particularly when comparing cultivars or species.

\section{REFERENCES}

Buxton, D.R., and S.L. Fales. 1994. Plant environment and quality. p. 155-199. In G.C. Fahey, Jr. et al. (ed.) Forage quality, evaluation, and utilization. ASA, CSSA, and SSSA, Madison, WI.

Buxton, D.R., and J.S. Hornstein. 1986. Cell-wall concentration and components in stratified canopies of alfalfa, birdsfoot trefoil, and red clover. Crop Sci. 26:180-184.

Buxton, D.R., J.S. Hornstein, W.F. Wedin, and G.C. Marten. 1985 Forage quality in stratified canopies of alfalfa, birdsfoot trefoil, and red clover. Crop Sci. 25:273-279.

Buxton, D.R., and G.C. Marten. 1989. Forage quality of plant parts of perennial grasses and relationships to phenology. Crop Sci. 29:429-435.

Chacon, E.A., and T.H. Stobbs. 1976. Influence of progressive defoliation of a grass sward in the eating behaviour of cattle. Aust. J. Agric. Res. 27:709-727.

Chacon, E.A., T.H. Stobbs, and M.B. Dale. 1978. Influence of sward characteristics on grazing behaviour and growth of Hereford steers grazing tropical grass pastures. Aust. J. Agric. Res. 29:89-102.

Fick, G.W., P.W. Wilkens, and J.H. Cherney. 1994. Modeling forage quality changes in the growing crop. p. 757-795. In G.C. Fahey, Jr., et al. (ed.) Forage quality, evaluation, and utilization. ASA, CSSA, and SSSA, Madison, WI.

Forbes, T.D.A., and S.W. Coleman. 1993. Forage intake and ingestive behavior of cattle grazing old world bluestems. Agron. J. 85:808-816.

Frank, A.B. 1991. Morphological development of crested and western wheatgrass following grazing. Agron. J. 83:826-828.

Frank, A.B., and V.B. Cardwell. 1997. Growth staging in research and crop management. Crop Sci. 37:1039-1040.

Frank, A.B., and L. Hofmann. 1989. Relationship among grazing management, growing degree days, and morphological development for native grasses on the Northern Great Plains. J. Range Manage. 42:199-202.

Gabrielsen, B.C., K.P. Vogel, B.E. Anderson, and J.K. Ward. 1990. Alkali-labile cell-wall phenolics and forage quality in switchgrass selected for differing digestibility. Crop Sci. 30:1313-1320.

Kalu, B.A., and G.W. Fick. 1981. Quantifying morphological stage of development of alfalfa for studies of herbage quality. Crop Sci. 21:267-271.

Mitchell, R., J. Fritz, K. Moore, L. Moser, K. Vogel, D. Redfearn, and D. Wester. 2001. Predicting forage quality in switchgrass and big bluestem. Agron. J. 93:118-124.

Mitchell, R.B., K.J. Moore, L.E. Moser, J.O. Fritz, and D.D. Redfearn. 1997. Predicting developmental morphology in switchgrass and big bluestem. Agron. J. 89:827-832. 
Moore, K.J., L.E. Moser, K.P. Vogel, S.S. Waller, B.E. Johnson, and J.F. Pedersen. 1991. Describing and quantifying growth stages of perennial forage grasses. Agron. J. 83:1073-1077.

Sanderson, M.A., and W.F. Wedin. 1989. Phenological stage and herbage quality relationships in temperate grasses and legumes. Agron. J. 81:864-869.

SAS Institute. 1990. SAS user's guide: Statistics. Version 6. SAS Inst., Cary, NC.

Simon, U., and B.H. Park. 1983. A descriptive scheme for stages of development in perennial forage grasses. p. 416-418. In J.A. Smith and V.W. Hays (ed.) Proc. Int. Grassl. Congr., 14th, Lexington, KY. 15-24 June 1981. Westview Press, Boulder, CO.

Vogel, K.P., J.F. Pedersen, S.D. Masterson, and J.J. Toy. 1999. Evaluation of a filter bag system for NDF, ADF, and IVDMD forage analysis. Crop Sci. 39:276-279.

West, C.P., D.W. Walker, R.K. Bacon, D.E. Longer, and K.E. Turner. 1991. Phenological analysis of forage yield and quality in winter wheat. Agron. J. 83:217-224.

\title{
Performance of Annual Medic Species (Medicago spp.) in Southeastern Wyoming
}

\author{
Michael J. Walsh,* Ronald H. Delaney, Robin W. Groose, and James M. Krall
}

\begin{abstract}
Annual medic (Medicago spp.) pastures that produce high levels of good quality forage are well suited to grazing and are used extensively throughout dryland farming regions of the world. In these regions, they are normally an integral component of cropping rotations because they allow for reductions in weed and disease problems in addition to increasing soil $\mathbf{N}$ levels for subsequent crops. The objective of this research was to investigate the performance of 17 annual medic cultivars and experimental lines for their potential use as self-regenerating annual pastures in the dryland cropping region of southeastern Wyoming. Dry matter and seed production capabilities were recorded over three seasons, 1996 to 1998. Growth phase development following different emergence times was evaluated in two seasons, and the forage quality was assessed for medic cultivars and lines grown in the 1997 season. Results revealed that the M. rigidula (L.) All. line, SA10343, consistently produced the greatest level of dry matter, with more than double the amount of forage than nearly all other cultivars. Dry matter production was related to the period of growth and development where higher yielding cultivars showed extended periods of vegetative growth. In general, the southeastern Wyoming climate substantially reduced the growth and development periods of medic cultivars bred in southern Australia. Given the overall performance of all cultivars, it was determined that the $M$. rigidula species had the greatest potential for further development in this environment.
\end{abstract}

$\mathrm{T}$ HE SYSTEM of integrated crop and livestock production known as ley farming was developed in the semiarid dryland farming regions of southern Australia. The ley-farming system is based on the rotation of cereal crops with annual legume pasture phases that regenerate from seed (self regenerating) at the start of each pasture phase. These pastures typically use annual medics as the legume pasture component (Crawford et al., 1989). The establishment of this system relies on the effectiveness of the pasture phase of the rotation in producing large amounts of high quality forage for graz-

M.J. Walsh, WAHRI, Faculty of Agric., Univ. of Western Australia, Nedlands, WA 6907, Australia; and R.H. Delaney, R.W. Groose, and J.M. Krall, Dep. of Plant Sci., Univ. of Wyoming, Laramie, WY 82070. Received 26 Apr. 2001. *Corresponding author (mwalsh@agric.uwa. edu.au).

Published in Agron. J. 93:1249-1256 (2001). ing. Additional requirements are that these pasture phases allow for the control of weeds and diseases while improving the structure and fertility of the soil. An additional criterion for these pastures is that they need to maintain a sufficiently high seedbank level to allow regeneration from seed at the start of each pasture phase. Annual medic pastures have been used for these purposes with considerable success for many years in Australia's dryland cropping regions (Puckridge and French, 1983). In these regions, annual medics are highly suited as a productive pasture phase despite harsh environmental conditions. The resulting reductions in weed and disease problems along with improved soil fertility and structure have led to a stable wheat (Triticum aestivum L.) production system.

As there are appropriate soil types and sufficient precipitation levels, it is believed that an annual medic based ley-farming system can be developed for use in southeastern Wyoming. Developing this farming system for this region relies on the inclusion of an annual medic pasture that is effective in producing increased levels of high quality forage over a summer growing season. Both the amount of $\mathrm{N}$ fixed and the additions to soil organic matter levels are directly related to dry matter production levels (Crawford et al., 1989). Additionally, seed production and, therefore, seedbank establishment for regeneration are also linked to the level of dry matter produced (Puckridge and French, 1983).

The differences in environmental conditions between a Mediterranean winter growing season and the summer growing season of southeastern Wyoming are likely to affect the growth and development of annual medics. There are several environmental factors, including temperature, moisture stress, and photoperiod that have been found to affect the growth and development of annual medics (Aitken, 1955; Clarkson and Russell, 1975, 1976; Van Heerden, 1984). These studies suggest that with longer photoperiods and higher temperatures, the development periods of annual medics are likely to be reduced in a summer growing season. Regardless, annual medics have proven to be highly adaptive to a wide range of environments and locations (Crawford et al., 1989).

Annual medic pasture regeneration depends on the 